\title{
In Vivo Characterization of Platinum(II)-Based Linker Technology for the Development of Antibody-Drug Conjugates: Taking Advantage of Dual Labeling with ${ }^{195 m P t}$ and ${ }^{89} \mathrm{Zr}$
}

\author{
Joey A. Muns ${ }^{1}$, Veronica Montserrat ${ }^{1}$, Hendrik-Jan Houthoff ${ }^{1}$, Karlijn Codée-van der Schilden ${ }^{2}$, Oene Zwaagstra ${ }^{2}$, \\ Niels J. Sijbrandi ${ }^{1}$, Eugen Merkul ${ }^{1}$, and Guus A.M.S. van Dongen ${ }^{3}$ \\ ${ }^{1}$ LinXis BV, Amsterdam, The Netherlands; ${ }^{2}$ Nuclear Research and Consultancy Group (NRG), Petten, The Netherlands; and \\ ${ }^{3}$ Department of Radiology and Nuclear Medicine, VU University Medical Center, Amsterdam, The Netherlands
}

\begin{abstract}
Linker instability and impaired tumor targeting can affect the tolerability and efficacy of antibody-drug conjugates (ADCs). To improve these ADC characteristics, we recently described the use of a metal-organic linker, [ethylenediamineplatinum(II)] $]^{2+}$, herein called $L x$. Initial therapy studies in xenograft-bearing mice revealed that trastuzumab- $L x$-auristatin $F(A F)$ outperformed its maleimide benchmark trastuzumab-mal-AF and the Food and Drug Administrationapproved ado-trastuzumab emtansine, both containing conventional linkers. In this study, we aimed to characterize $L x$-based ADCs

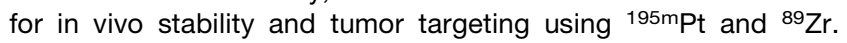
Methods: The $\gamma$-emitter ${ }^{195 m P t}$ was used to produce the radiolabeled $L x\left[{ }^{195 \mathrm{mPt}} \mathrm{Lx}\right.$. ${ }^{89} \mathrm{Zr}$-Desferrioxamine ( ${ }^{89} \mathrm{Zr}$-DFO) was conjugated to trastuzumab either via [195mPt] $L x$ (to histidine residues) or conventionally (to lysine residues) in order to monitor the biodistribution of antibody, payload, and linker separately. Linker stability was determined by evaluating the following ADCs for biodistribution in NCl-N87 xenograft-bearing nude mice $72 \mathrm{~h}$ after injection: trastuzumab-[ ${ }^{195 \mathrm{mPt}} \mathrm{L} x$-DFO- ${ }^{89} \mathrm{Zr}$, trastuzumab-[ ${ }^{195 \mathrm{mPt}]} \mathrm{Lx}-$ $\mathrm{AF}$, and ${ }^{89} \mathrm{Zr}$-DFO-(Lys)trastuzumab (control), all having drug-toantibody ratios (DARs) of 2.2-2.5. To assess the influence of DAR on biodistribution, ${ }^{89} \mathrm{Zr}$-DFO-(Lys)trastuzumab- $L x-A F$ with an AF-to-antibody ratio of $0,2.6$, or 5.2 was evaluated $96 \mathrm{~h}$ after injection. Results: Similar biodistributions were observed for trastuzumab-[195mPt] $L x$-DFO- ${ }^{89} \mathrm{Zr}$, trastuzumab-[195mPt] $L x-A F$, and ${ }^{89} \mathrm{Zr}$-DFO-(Lys)trastuzumab irrespective of the isotope used for biodistribution assessment. The fact that $L x$ follows the antibody biodistribution indicates that the payload- $L x$ bond is stable in vivo. Uptake of the 3 conjugates, as percentage injected dose (\%ID) per gram of tissue, was about $30 \% \mathrm{ID} / \mathrm{g}$ in tumor tissue but less than $10 \%$ ID/g in most healthy tissues. Trastuzumab-[195mPt] $L x-A F$ (DAR 2.2) showed a tendency toward faster blood clearance and an elevated liver uptake, which increased significantly to $28.1 \pm 4.2 \% \mathrm{ID} / \mathrm{g}$ at a higher DAR of 5.2, as revealed from the biodistribution and PET

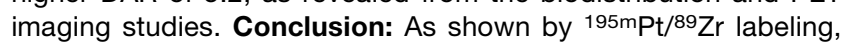
ADCs containing the $L x$ linker are stable in vivo. In the case of trastuzumab- $L x-A F$ (DARs 2.2 and 2.6), an unimpaired biodistribution was demonstrated.
\end{abstract}

Received Dec. 5, 2017; revision accepted Feb. 3, 2018.

For correspondence or reprints contact: Niels J. Sijbrandi, LinXis BV, De Boelelaan 1085c, Amsterdam, $1081 \mathrm{HV}$, The Netherlands.

E-mail: sijbrandi@linxispharmaceuticals.com

Published online Mar. 1, 2018.

COPYRIGHT (C 2018 by the Society of Nuclear Medicine and Molecular Imaging.
Key Words: ADC radiolabeling; $L x$ linker; antibody-drug conjugates; ${ }^{195 \mathrm{mPt}}{ }^{89} \mathrm{Zr}$

J Nucl Med 2018; 59:1146-1151

DOI: $10.2967 /$ jnumed.117.206672

$\mathbf{F}$ our antibody-drug conjugates (ADCs) have been approved by the Food and Drug Administration (Adcetris [brentuximab vedotin; Seattle Genetics] (1), Kadcyla [ado-trastuzumab emtansine; Genentech] (2), Mylotarg [gemtuzumab ozogamicin; Pfizer], and Besponsa [inotuzumab ozogamicin; Pfizer] (3)), and more than 70 are currently under clinical evaluation (4). Nevertheless, approvals are stagnating, and several ADCs have failed to be approved because of safety issues or insufficient efficacy (5). Therefore, a deeper insight into the in vivo performance of ADCs is clearly desirable.

The linker system used for coupling of the drug to the monoclonal antibody is of key importance for efficacy but also for the tolerability and safety of an ADC (6-9). First, the drug can be released from the monoclonal antibody into the circulation, resulting in unwanted sequestration of the drug in healthy tissues $(10,11)$. Second, the antibody itself can be affected by drug conjugation, especially at a high drug-to-antibody ratio (DAR), resulting in impaired tumor binding or faster blood clearance of the ADC, with uptake in catabolic organs such as liver and spleen $(12,13)$. Third, after cellular uptake of an ADC and subsequent catabolism, the drug can become detached from the antibody and eventually be released from the cell $(5,14-16)$. It can subsequently kill neighboring cancer cells (bystander effect), but it can also result in increased toxicity by systemic exposure.

Most linker systems currently used in clinical studies are based on conventional active ester or maleimide chemistry for drug conjugation to lysine or cysteine residues of the monoclonal antibody, respectively. It has been recognized that these linkers provide suboptimal ADCs; therefore, extensive research on new conjugation technologies has been initiated during the last few years (5,17-19). As a pioneering approach in the development of ADCs, we recently described the use of the cationic metal-organic linker [ethylenediamineplatinum(II)] ${ }^{2+}$, herein called $L x$ (LinXis) $(20,21)$. In a first step, $L x$ can be coordinated to payloads bearing nonconventional functionalities, such as an $N$-heterocyclic ligand, to provide storable products that we term semifinal. In a second step, an 
$L x$-drug semifinal product is conjugated efficiently to histidine residues of unmodified monoclonal antibodies. On the basis of these characteristics, the $L x$ linker technology can pave the way to a plugand-play ADC development platform in which antibodies and payloads can easily be varied. The potential of the $L x$ linker technology was recently demonstrated in the preparation of auristatin $\mathrm{F}$ conjugated trastuzumab (trastuzumab- $L x$-AF) (21). A single dose of zumab-mal-AF and the Food and Drug Administration-approved ado-trastuzumab emtansine in a xenograft mouse model of gastric cancer (NCI-N87) and of ado-trastuzumab emtansine-resistant breast cancer (JIMT-1). Nevertheless, an in-depth analysis of the in vivo performance of $L x$-based ADCs is needed to disclose the distinguished properties of $L x$ in more detail.

In general, for the assessment of in vivo stability and tumor targeting of an ADC, tissue samples are taken for analytic evaluation of ADC integrity and uptake, for example, by enzyme-linked immunosorbent type assays or liquid chromatography-mass spectrometry/ mass spectrometry (22-25). However, such procedures are invasive, time consuming, difficult to apply for longitudinal whole-body analysis including tumor uptake analysis, and inaccurate with respect to quantification. trastuzumab- $L x$-AF outperformed its maleimide benchmark trastu-

For the in vivo characterization of $L x$-based ADCs, we therefore present an alternative approach that exploits an integrated strategy of dual radiolabeling and immuno-PET imaging using the radionuclides ${ }^{89} \mathrm{Zr}$ and ${ }^{195 \mathrm{mPt}}$. In previous studies, we have demonstrated how ${ }^{89} \mathrm{Zr}$-immuno-PET can be used for the in vivo evaluation of biologicals, including ADCs (26-28). For this purpose, we have developed generic good-manufacturing-practice-compliant labeling methods using the bifunctional chelator desferrioxamine (DFO) (Desferal; Novartis) for coupling of ${ }^{89} \mathrm{Zr}$ to antibodies $(29,30)$. Here, we show for the first time how ${ }^{195 \mathrm{~m}} \mathrm{Pt}$, produced in the high-flux reactor in Petten (Supplemental Table 1 shows the batch characteristics of the delivered ${ }^{195 \mathrm{~m} P t-c o m p l e x}$; supplemental materials are available at http://jnm.snmjournals.org), can be used to obtain the radioactive

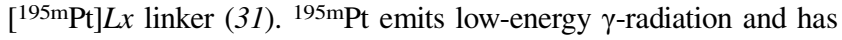
a half-life of $4.02 \mathrm{~d}$, which matches the biologic half-life of antibodies. The combination of ${ }^{195 \mathrm{mPt}}$ and ${ }^{89} \mathrm{Zr}$ allows sensitive and direct detection of the $L x$ linker next to the antibody and the drug payload (for this purpose, ${ }^{89} \mathrm{Zr}$-DFO was used as a model) at low concentrations in tissue samples. The radiolabels ${ }^{89} \mathrm{Zr}$ and ${ }^{195 \mathrm{~m} P t}$ were exploited to demonstrate the in vivo stability of $L x$-based ADCs and the in vivo uptake and retention of $L x$-based ADCs in tumors and healthy tissues in relation to DAR. To characterize $L x$ as an ADC linker in vivo, ${ }^{89} \mathrm{Zr}$-DFO was conjugated to trastuzumab either via [ $\left.{ }^{195 \mathrm{mPt}}\right] L x$ (to histidine residues) or conventionally (to lysine residues). The following constructs were evaluated in comparative biodistribution and imaging studies: trastuzumab-[ $\left.{ }^{195 \mathrm{mPt}}\right] L x$ DFO- ${ }^{89} \mathrm{Zr}$, trastuzumab-[ ${ }^{195 \mathrm{mPt}]} L x-\mathrm{AF},{ }^{89} \mathrm{Zr}-$ DFO-(Lys)trastuzumab- $L x$-AF, and ${ }^{89} \mathrm{Zr}$ DFO-(Lys)trastuzumab (control).

\section{MATERIALS AND METHODS}

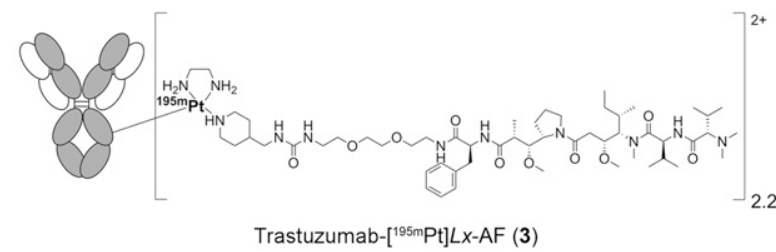

B

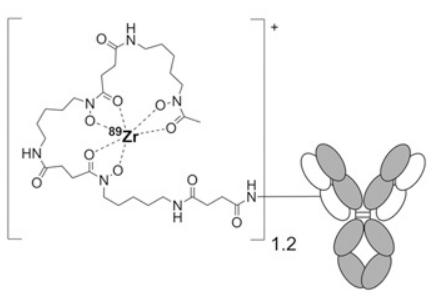

${ }^{89} \mathrm{Zr}$-DFO-trastuzumab (4)

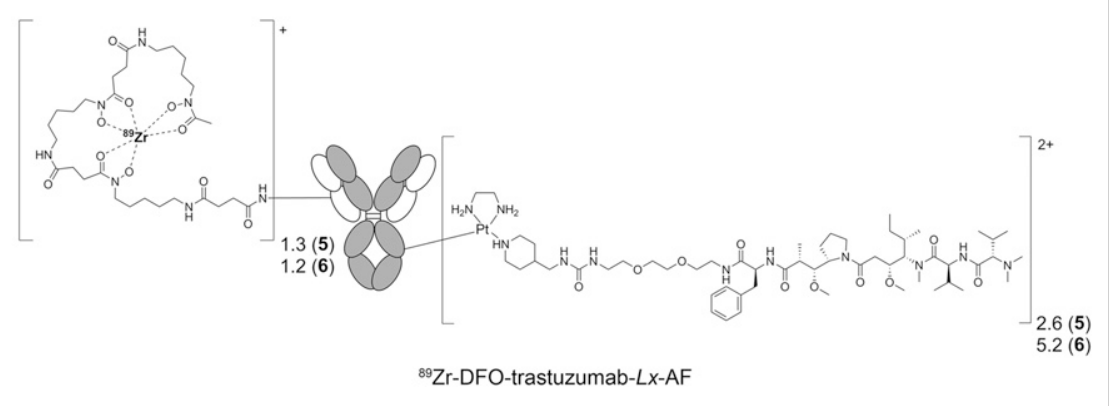

FIGURE 1. Structural representation of constructs 1-6.
Cell Lines and General Materials and Procedures

This information is available from the authors on request.

\section{Synthesis of [195mPt]Lx-Based Conjugates}

Details on the chemical structures, synthesis, and analysis of constructs 1-3 (Fig. 1A) used for in vivo evaluation are available from the authors on request.

\section{Synthesis of ${ }^{89} \mathrm{Zr}$-DFO-(Lys) Trastuzumab- $L x-A F$ with Different AF-to-Antibody Ratios}

Details on the synthesis of ${ }^{89} \mathrm{Zr}$-DFO-(Lys) trastuzumab- $L x$-AF with AF-to-antibody ratios of $2.6(5)$ and $5.2(6)$ (Fig. 1B) are available from the authors on request. As a control for biodistribution and imaging studies, ${ }^{89} \mathrm{Zr}$ DFO-(Lys)trastuzumab (4) (Fig. 1B), synthesized as described previously (29), was included.

\section{Analytic Procedures}

Procedures to assess the conjugation efficiency and the DAR and to analyze the quality of the radiolabeled conjugates are available from the authors on request. 
TABLE 1

Characteristics of Radiolabeled Constructs 1-6 Evaluated in Tumor-Bearing Mice

\begin{tabular}{|c|c|c|c|c|c|}
\hline \multirow[b]{2}{*}{ Construct } & \multicolumn{2}{|c|}{ DAR } & \multicolumn{3}{|c|}{ Injected dose } \\
\hline & DFO & AF & Construct dose (mg/kg) & $89 \mathrm{Zr}(\mathrm{kBq})$ & $195 \mathrm{mPt}(\mathrm{kBq})$ \\
\hline Trastuzumab-[195mPt] $L x$-DFO-89 Zr (1) & 2.4 & - & 4 & 37.5 & 1.95 \\
\hline 89ZZr-DFO-(Lys)trastuzumab (2) & 2.5 & - & 4 & 460 & - \\
\hline Trastuzumab-[195mPt]Lx-AF (3) & - & 2.2 & 4 & - & 1.81 \\
\hline 89Zr-DFO-(Lys)trastuzumab (4) & 1.2 & - & 1 & $1.85 \times 10^{3}$ & - \\
\hline 89Zr-DFO-(Lys)trastuzumab-Lx-AF (5) & 1.3 & 2.6 & 1 & $1.95 \times 10^{3}$ & - \\
\hline${ }^{89} \mathrm{Zr}$-DFO-(Lys)trastuzumab-Lx-AF (6) & 1.2 & 5.2 & 1 & $1.74 \times 10^{3}$ & - \\
\hline
\end{tabular}

\section{Dual-Isotope ${ }^{195 m P t}$ and ${ }^{89} \mathrm{Zr}$ Counting}

To determine the optimal energy windows for simultaneous ${ }^{195 \mathrm{mPt}}$ and ${ }^{89} \mathrm{Zr}$ counting, the energy spectrums of both isotopes were assessed using an automatic $\gamma$-counter (Hidex). The energy windows for counting were set at $30-210 \mathrm{keV}$ and $400-1,100 \mathrm{keV}$ for ${ }^{195 \mathrm{mPt}}$ and ${ }^{89} \mathrm{Zr}$, respectively. To determine the activity of ${ }^{195 \mathrm{~m} P t}$ in the dualisotope experiments, the activity measured in the $30-210 \mathrm{keV}$ energy window needed to be corrected for the activity related to ${ }^{89} \mathrm{Zr}$ in this energy window using Equation 1:

$$
\operatorname{cpm}\left({ }^{195 \mathrm{~m}} \mathrm{Pt}\right)=\operatorname{cpm}_{(30-210 \mathrm{keV})}-C \times \mathrm{cpm}_{(400-1,100 \mathrm{keV})},
$$

in which

$$
C=\frac{\operatorname{cpm}_{\left({ }^{89} \mathrm{Zr} \mathrm{standard}, 30-210 \mathrm{keV}\right)}}{\operatorname{cpm}_{\left({ }^{89} \mathrm{Zr} \mathrm{standard}, 400-1,100 \mathrm{keV}\right)}}
$$

Since ${ }^{195 \mathrm{mPt}}$ does not emit photons in the ${ }^{89} \mathrm{Zr}$ energy window, the activity measured in this window can be directly related to ${ }^{89} \mathrm{Zr}$ using Equation 2:

$$
\operatorname{cpm}\left({ }^{89} \mathrm{Zr}\right)=\operatorname{cpm}_{(400-1,100 \mathrm{keV})} .
$$

\section{Biodistribution of [ ${ }^{195 \mathrm{~m} P t}$ Lx-Based Conjugates}

The biodistribution of constructs 1-3 was evaluated in nude mice bearing the human epidermal growth factor receptor 2-positive gastric cancer xenograft line NCI-N87. All animal experiments were performed according to the National Institutes of Health Principles of Laboratory Animal Care and Dutch national law ("Wet op de dierproeven," Staatsblad 1985, 336). Nineteen days before administration of the constructs, 19 female mice (HSD:Athymic Nude-Foxn1nu, 21-31 g [Harlan]; 8-10 wk old at the time of the experiments) were injected subcutaneously with $2.5 \times 10^{6}$ NCI-N87 cells in both flanks. Mice bearing NCI-N87 xenografts (tumor volume $100-250 \mathrm{~mm}^{3}$ ) were anesthetized by inhalation of $2 \%$ isoflurane and injected intravenously via the retroorbital plexus (32) with the radiolabeled constructs $(\sim 4 \mathrm{mg} / \mathrm{kg}$ for ADCs 1-3) in a $100 \mu \mathrm{L}$ injection volume. The injected ${ }^{195 \mathrm{~m}} \mathrm{Pt}$ and ${ }^{89} \mathrm{Zr}$ radioactivity doses were chosen in such a way that an accurate correction of the ${ }^{195 \mathrm{mPt}}$ signal in the $\gamma$-counter for the contribution of ${ }^{89} \mathrm{Zr}$ counts was possible. These requirements were crucial during synthesis and radiolabeling and resulted in the samples for injection as presented in Table 1.

Blood was collected via the tail 2, 24, and $48 \mathrm{~h}$ after injection of the tracer. At the end of the experiment, $72 \mathrm{~h}$ after injection, the mice were anesthetized, bled, sacrificed, and dissected. After blood, tumor, and healthy tissues had been weighed, the amount of radioactivity in each sample was measured in a $\gamma$-counter for each radioisotope present. Radioisotope uptake was calculated as the percentage of the injected dose per gram of tissue (\%ID).

\section{Biodistribution of ${ }^{89} \mathrm{Zr}$-Labeled $L x$-Based ADCs with Different AF-to-Antibody Ratios}

The biodistribution of ${ }^{89} \mathrm{Zr}$-DFO-(Lys)trastuzumab- $L x$-AF constructs 5 and $\mathbf{6}$ with different AF-to-antibody ratios was evaluated in NCI-N87 tumor-bearing mice and compared with the biodistribution of ${ }^{89} \mathrm{Zr}$ DFO-(Lys)trastuzumab (4). All conjugates (Fig. 1B) were prepared with a relatively low ${ }^{89} \mathrm{Zr}$-DFO-to-antibody ratio of approximately 1 (Table 1) to minimize a potential contribution of ${ }^{89} \mathrm{Zr}$-DFO to pharmacokinetic effects.

After administration of the conjugates, PET imaging was performed with a dedicated small-animal NanoPET/CT scanner (Mediso Ltd.). A mouse of each group was anesthetized by inhalation of $2 \%$ isoflurane and scanned $96 \mathrm{~h}$ after injection for $1 \mathrm{~h}$. A CT scan was acquired before the PET scan and used for attenuation and scatter correction. Reconstruction was performed with a fully 3-dimensional algorithm (Tera-Tomo; Mediso Ltd.) with 4 iterations and 6 subsets, resulting in an isotropic $0.4-\mathrm{mm}$ voxel dimension.

\section{Statistical Analysis}

All animal experiments were statistically analyzed using the Welch $t$ test for independent samples. Two-sided significance levels were calculated, and a $P$ value of less than 0.05 was considered statistically significant.

\section{RESULTS}

\section{Rationale for Preparation of Radiolabeled Constructs}

Three constructs (Fig. 1A) were prepared to allow characterization of the $L x$ linker in biodistribution studies. For this purpose, either the diagnostic payload ${ }^{89} \mathrm{Zr}$-DFO or the therapeutic payload AF was coupled to trastuzumab via $\left[{ }^{195 \mathrm{mPt}}\right] L x$ (Table 1). By assessment of ${ }^{195 m P t}$ and ${ }^{89} \mathrm{Zr}$ counts, crucial information on the $L x$ in vivo performance was expected to be obtained from the following constructs: trastuzumab-[ $\left.{ }^{195 \mathrm{~m} P t}\right] L x$-DFO- ${ }^{89} \mathrm{Zr}(\mathbf{1}),{ }^{89} \mathrm{Zr}-\mathrm{DFO}-$ (Lys)trastuzumab (2), and trastuzumab-[195mPt] $L x-\mathrm{AF}(\mathbf{3})$.

The evaluation of conjugate $\mathbf{1}$ gives information on the stability of $L x$-based ADCs in blood and at the tumor site and on sequestration of putative $L x$-containing metabolites along the body, as is important for the tolerability of $L x$-based ADCs. Subsequently, the comparison of conjugates $\mathbf{1}$ and $\mathbf{2}$ provides information about stability of the metalorganic linker $L x$ compared with a classic covalent organic linker. Further, the comparison of conjugates $\mathbf{1}$ and $\mathbf{3}$ allows determination of the biodistribution and tumor uptake of $L x$-based ADCs related to the type of payload: the diagnostic moiety ${ }^{89} \mathrm{Zr}$-DFO or the therapeutic drug AF. Finally, ${ }^{89} \mathrm{Zr}$-labeled AF-bearing constructs 5 and $\mathbf{6}$ along with an AF-free reference construct 4 (Fig. 1B), are used to evaluate the effect of AF-to-antibody ratio on the biodistribution. 


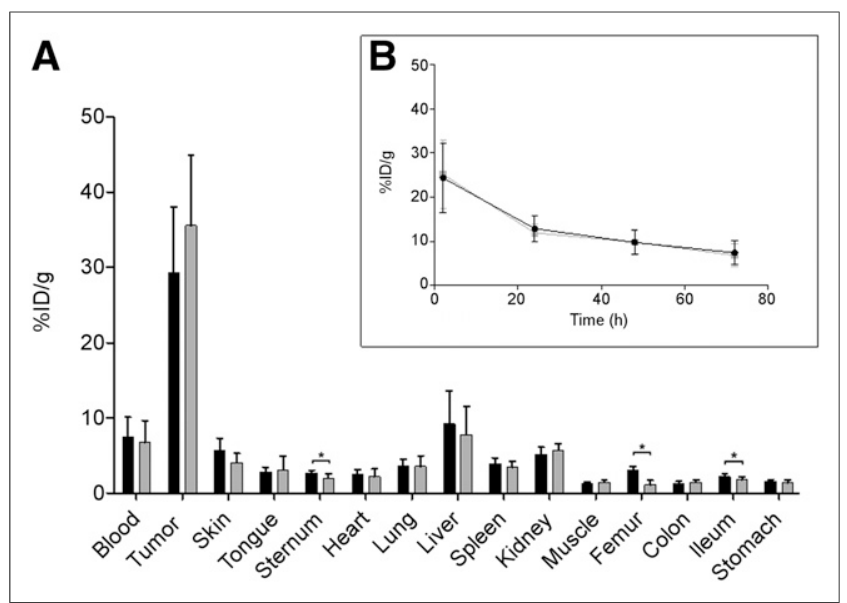

FIGURE 2. Biodistribution $72 \mathrm{~h}$ after injection (A) and blood kinetics (B) of $4 \mathrm{mg} / \mathrm{kg}$ dose of trastuzumab-[195mPt] $L x$-DFO-89Zr (1, DAR $2.4 ; n=7$ ) in NCl-N87 xenograft-bearing mice. Black bars and line $={ }^{89} \mathrm{Zr}$ counting; gray bars and line $=195 \mathrm{mPt}$ counting

\section{Biodistribution of [ ${ }^{195 m P t}$ Lx -Based Conjugates}

Biodistribution of conjugates 1-3 was assessed in $n u / n u$ mice bearing NCI-N87 xenografts at $72 \mathrm{~h}$ after injection. All tissue and blood values are presented in Supplemental Tables 2-7.

Biodistribution of Trastuzumab- $\left[{ }^{195 m} \mathrm{Pt}\right] L x-D F O-{ }^{89} \mathrm{Zr}$ (1). Biodistribution analysis of trastuzumab-[195m Pt] $L x$-DFO- ${ }^{89} \mathrm{Zr}$ (1) revealed similar levels of ${ }^{195 \mathrm{~m}} \mathrm{Pt}$ and ${ }^{89} \mathrm{Zr}$ in blood, tumors, and most of the healthy tissues (Fig. 2A). In sternum and femur, a higher uptake of ${ }^{89} \mathrm{Zr}$ than of ${ }^{195 \mathrm{mPt}}$ was found, as can be explained by minor instability of the ${ }^{89} \mathrm{Zr}$-DFO complex and the osteophilic properties of unbound ${ }^{89} \mathrm{Zr}$ (33). Similar blood levels were confirmed by analysis of blood kinetics (Fig. 2B). These results confirm the in vivo stability of the coordinative bond between the $L x$ linker and the payload.

Biodistribution of Trastuzumab- $\left[{ }^{195 m} \mathrm{Pt}\right] \mathrm{Lx}-\mathrm{DFO}-{ }^{89} \mathrm{Zr}$ (1) Versus ${ }^{89} \mathrm{Zr}$-DFO-(Lys)trastuzumab (2). The biodistribution of trastuzumab[ $\left.{ }^{195 \mathrm{mPt}}\right] L x$-DFO- ${ }^{89} \mathrm{Zr}$ (1) was compared with the biodistribution of ${ }^{89} \mathrm{Zr}$-DFO-(Lys)trastuzumab (2) on the basis of ${ }^{89} \mathrm{Zr}$ counting (Fig. 3A). Both constructs demonstrated a similar biodistribution irrespective of whether ${ }^{89} \mathrm{Zr}$-DFO was conjugated via $\left[{ }^{195 \mathrm{mPt}}\right] L x$ to histidine residues or via classic conjugation to the lysine residues of the antibody. Nevertheless, a faster blood clearance of the $L x$-based construct was observed, accompanied by a slightly increased liver uptake. The tendency for a faster blood clearance was confirmed by pharmacokinetic analyses (Fig. 3B). The combined results of Figures 2 and 3 show that the biodistribution of the $\left[{ }^{195 \mathrm{~m}} \mathrm{Pt}\right] L x$ linker follows the biodistribution of the antibody, indicating that $L x$-based ADCs are stable in vivo. Nevertheless, conjugation to histidine residues instead of conjugation to lysine residues slightly alters the pharmacokinetics.

Biodistribution of Trastuzumab- $\left[{ }^{195 m} \mathrm{Pt}\right] L x-D F O-{ }^{89} \mathrm{Zr}$ (1) Versus Trastuzumab- $\left[{ }^{195 m} P t\right] L x-A F(3)$. The biodistribution of trastuzumab[195mPt] $L x$-DFO- ${ }^{89} \mathrm{Zr}(\mathbf{1})$ and trastuzumab-[195mPt] $L x$-AF (3) was compared on the basis of ${ }^{195 m P t}$ counting (Fig. 4A). Despite the different payloads, ${ }^{89} \mathrm{Zr}$-DFO or AF, both conjugates showed similar blood levels, tumor uptake, and uptake in most of the dissected tissues. Only in the liver did trastuzumab-[195mPt] $L x$-AF (3) show a higher uptake than trastuzumab-[ $\left.{ }^{195 \mathrm{mPt}}\right] L x$-DFO- ${ }^{89} \mathrm{Zr}$ (1): $17.0 \pm$ $2.2 \% \mathrm{ID} / \mathrm{g}$ versus $7.8 \pm 3.8 \% \mathrm{ID} / \mathrm{g}$, respectively. Similar blood levels were confirmed by pharmacokinetic analyses (Fig. 4B). These results

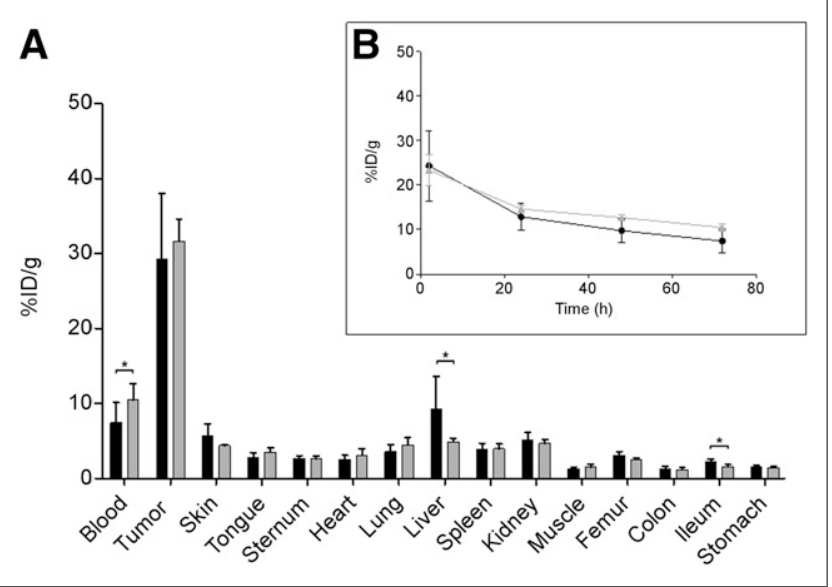

FIGURE 3. Biodistribution $72 \mathrm{~h}$ after injection (A) and blood kinetics (B) of $4 \mathrm{mg} / \mathrm{kg}$ dose of trastuzumab-[195mPt]Lx-DFO-89Zr (1, DAR 2.4; $n=7$ ) and ${ }^{89} \mathrm{Zr}$-DFO-(Lys)trastuzumab (2, DAR $2.5 ; n=6$ ) in NCl-N87 xenograft-bearing mice, both assessed by ${ }^{89} \mathrm{Zr}$ counting. Black bars and line $=$ trastuzumab-[195mPt] $L x-D F O-{ }^{89} \mathrm{Zr}$ (1); gray bars and line $={ }^{89} \mathrm{Zr}-\mathrm{DFO}-$ (Lys)trastuzumab (2).

indicate that $L x$-based ADCs are stable in vivo irrespective of the nature of the payload but that the nature of the payload might affect the biodistribution.

\section{Biodistribution of ${ }^{89} \mathrm{Zr}$-DFO-(Lys)trastuzumab-Lx-AF with Different AF-to-Antibody Ratios}

We found that, compared with the ADC containing ${ }^{89} \mathrm{Zr}-\mathrm{DFO}$ as the payload, coupling of AF to histidine residues of trastuzumab via $L x$ with a DAR of 2.2 caused slightly faster clearance of the ADC from the blood, accompanied by a higher liver uptake (Fig. 4). This finding indicates that a DAR of around 2.2 might be the maximum for optimal tumor targeting. To explore this assumption, ${ }^{89} \mathrm{Zr}$-DFO-(Lys)trastuzumab- $L x$-AF conjugates with an AF-toantibody ratio of $0(4), 2.6(5)$, and $5.2(6)$ were evaluated in biodistribution (Fig. 5A) and PET imaging studies (Fig. 5C) in mice

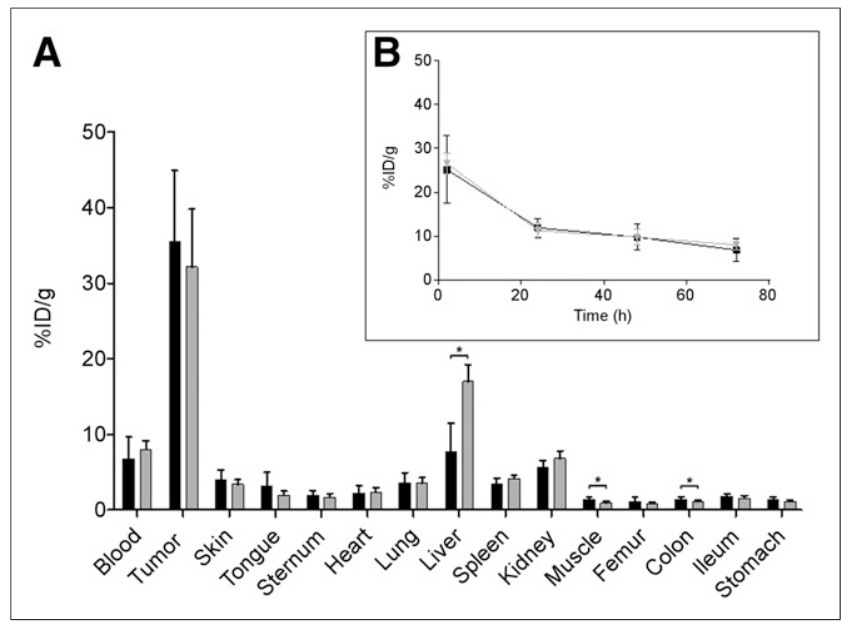

FIGURE 4. Biodistribution $72 \mathrm{~h}$ after injection $(\mathrm{A})$ and blood kinetics $(\mathrm{B})$ of $4 \mathrm{mg} / \mathrm{kg}$ dose of trastuzumab-[195mPt]Lx-DFO-89Zr (1, DAR 2.4; $n=7$ ) and trastuzumab-[195mPt]Lx-AF (3, DAR 2.2; $n=6)$ in NCI-N87 xenograft-bearing mice, both assessed by ${ }^{195 \mathrm{~m}} \mathrm{Pt}$ counting. Black bars and line $=$ trastuzumab- $\left[{ }^{195 \mathrm{~m} P t}\right] \angle x-D F O-{ }^{89} \mathrm{Zr}(\mathbf{1})$; gray bars and line $=$ trastuzumab-[195mPt]Lx-AF (3). 


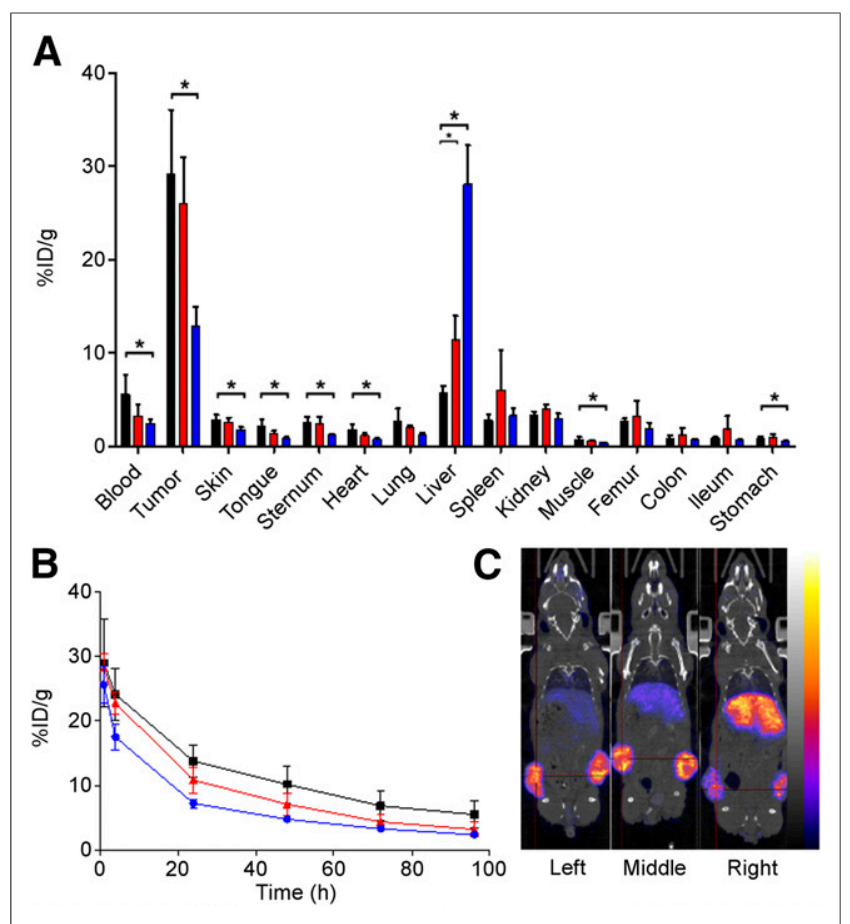

FIGURE 5. Biodistribution $96 \mathrm{~h}$ after injection (A), blood kinetics (B), and PET images $96 \mathrm{~h}$ after injection (C) of ${ }^{89} \mathrm{Zr}$-DFO-trastuzumab- $L x-A F$, with AF-to-antibody ratios of 0 (4), 2.6 (5), and 5.2 (6), to NCI-N87 xenograft-bearing mice. Black bars, black line, and left PET image = AF-to-antibody ratio of 0 (4); red bars, red line, and middle PET image = AF-to-antibody ratio of 2.6 (5); blue bars, blue line, and right PET image $=$ AF-to-antibody ratio of $5.2(6)$.

bearing NCI-N87 xenografts $96 \mathrm{~h}$ after injection. In addition, blood kinetics (Fig. 5B) were assessed up to $96 \mathrm{~h}$ after injection.

Conjugate 6, with the highest DAR (5.2), demonstrated impaired tumor targeting, faster blood clearance, and increased liver uptake (Fig. 5A) than conjugate 4 (DAR 0), as was confirmed by the analysis of blood kinetics (Fig. 5B) and by PET imaging (Fig. 5C). Also, conjugate 5 (DAR 2.6) showed such a tendency, although no statistical differences were observed compared with conjugate 4 (Fig. 5). All tissue and blood values are presented in Supplemental Tables 8 and 9.

\section{DISCUSSION}

Recently, we introduced $L x$ as a promising metal-organic ADC linker $(20,21)$. In a first step, $L x$ is coordinated to diagnostic or therapeutic payloads to provide storable Pt(II) complexes, or semifinal products. In a second step, such an $L x$-payload complex is conjugated to an antibody, followed by a posttreatment step with thiourea to remove all weakly bound platinum complexes. Extensive in vitro analysis using ${ }^{89} \mathrm{Zr}$-DFO and AF as the payloads revealed that about $85 \%$ of an $L x$-payload species binds to the Fc region, presumably to histidines. The formed ADCs were found to be stable in phosphate-buffered saline and human serum without loss of the antibody binding affinity, and preliminary in vivo studies with trastuzumab- $L x$-DFO- ${ }^{89} \mathrm{Zr}$ indicated pharmacokinetics and tumor-targeting properties similar to the parental trastuzumab. Finally, trastuzumab- $L x$-AF appeared to be remarkably effective in therapy studies with NCI-N87 and JIMT-1 xenograft-bearing nude mice, a reason we aimed for a further disclosure of the distinguished properties of $L x$ in vivo.

In the current study, we explored a unique possibility arising

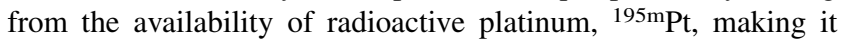

feasible to evaluate the in vivo performance of $L x$ in an accurate and facile way by radiolabeling each of the ADC components: the antibody (by ${ }^{89} \mathrm{Zr}$-DFO coupling to lysine residues of trastuzumab), the linker (using [ ${ }^{195 \mathrm{mPt}} \mathrm{L} x$ ), and the payload (using ${ }^{89} \mathrm{Zr}-\mathrm{DFO}$ as an exemplary diagnostic payload). Counting of the radioisotopes of 2 ADCs, trastuzumab-[ $\left.{ }^{195 \mathrm{~m} P t}\right] L x$-DFO- ${ }^{89} \mathrm{Zr}$ (1) and ${ }^{89} \mathrm{Zr}$-DFO-(Lys) trastuzumab (2), in biodistribution studies in NCI-N87 xenograftbearing nude mice revealed a similar biodistribution (Figs. 2 and 3). This was also the case when a therapeutic payload (AF) was used (Fig. 4). Therefore, it can be concluded that the $L x$-based ADCs are stable in serum and suitable for optimal tumor targeting. Results also revealed that $L x$ follows the biodistribution of an antibody and, unlike platinum-based chemotherapeutics such as cisplatin, remains an inert component of the ADC after in vivo administration during the time frame of the investigation. This information is important with respect to the tolerability and safety of $L x$-based ADCs.

Comparative biodistribution studies revealed a slightly faster blood clearance of the $L x$-based constructs, accompanied by an increased liver uptake (Fig. 3A). This effect became significant when the DAR of the $L x$-based constructs was increased from 2.6 to 5.2 (Fig. 5). Although we and others have previously described the phenomenon of increased blood clearance in relation to DAR $(12,34)$, it seems that $L x$ contributes to this phenomenon. One explanation may be an alteration of the antibody binding to the neonatal $\mathrm{Fc}$ receptor, which is known to be involved in recycling of IgGs and extension of their half-life in circulation (35). Histidines that are present on the $\mathrm{Fc}$ part of the antibody play an important role in neonatal Fc receptor binding of IgGs, and because $L x$ most probably conjugates to histidines, mainly in the $\mathrm{Fc}$ region (21), it is possible that $L x$ conjugation affects neonatal $\mathrm{Fc}$ receptor binding and the pharmacokinetics of a monoclonal antibody (36). Besides this $L x$-related effect, impaired biodistribution is dependent on the number and nature of payloads, which can cause alterations in hydrophobicity, charge, or conformation.

The biodistribution studies presented here provide strong evidence that premature release of the drug or of the $L x$-drug species in the blood does not occur, thus promising safety advantages for $L x$-based ADCs. A second way by which toxic metabolites may give safety issues, however, is by intracellular catabolism of an ADC and release of the metabolites into the circulation. Because of the proven stability of the $L x$ linker, we anticipate that the drug becomes released intracellularly as a His- $L x$-drug complex. After apoptosis, this metabolite may be released from the targeted cell and may enter the bloodstream (15). If such a metabolite is potent, it might cause systemic toxicity. In our previous study, we already tested the potency of $L x$-AF versus AF-mal in viability assays in vitro (21). In 6 tested cell lines, the potency of $L x$-AF appeared $10^{3}-10^{4}$ times lower than its corresponding $\mathrm{ADC}$, whereas for the maleimide-based linker and its corresponding ADC, the difference was only 10-100 times. We believe that the low toxicity of the $L x$-drug complex is due to the positive charge on platinum, which impedes passage through the cell membrane. The present and previous results indicate a favorable safety profile for $L x$-based ADCs. As a prelude to clinical studies, $L x$-based ADCs will be rigorously evaluated in vivo in preclinical safety studies and in metabolite studies using ${ }^{195 m P t}$.

The combined use of ${ }^{89} \mathrm{Zr}$ and ${ }^{195 \mathrm{~m} P t}$ in biodistribution and imaging experiments as described here appears to be a powerful tool for the preclinical in vivo characterization of $L x$-based ADCs. As demonstrated by us and others, in vivo characterization of antibodies and antibody conjugates such as ADCs by ${ }^{89} \mathrm{Zr}$-immuno-PET imaging can easily be translated to the clinic $(26,27)$. By assessment of selective 
tumor targeting at sufficiently high uptake levels, ${ }^{89} \mathrm{Zr}$-immuno-PET imaging of an ADC or its antibody component might be used for personalized treatment of tumors $(37-41)$. In contrast, at its current stage of development this is not yet possible by ${ }^{195 \mathrm{~m} P t}$ imaging. The amounts of platinum used in $L x$-based ADCs and the specific activity of ${ }^{195 \mathrm{~m} P t}$ are too low for preclinical or clinical SPECT imaging.

\section{CONCLUSION}

Radiochemistry in combination with PET imaging is a powerful tool for ADC development and in vivo characterization. ADCs containing the $L x$ linker are stable in vivo, and in the case of trastuzumab- $L x$-AF with DARs of 2.2 and 2.6, they showed an unimpaired biodistribution. $L x$ follows the biodistribution of the antibody, indicating that it remains an inert component of an ADC after its in vivo administration. This information is important with respect to the performance, tolerability, and safety of $L x$-based ADCs.

\section{DISCLOSURE}

Joey Muns, Veronica Montserrat, Niels Sijbrandi, and Eugen Merkul are employed by LinXis B.V.; Hendrik-Jan Houthoff is chief executive officer of LinXis B.V. and has ownership (including patents) in LinXis B.V.; Guus van Dongen is a member of the nonprofit scientific advisory board of LinXis B.V. No other potential conflict of interest relevant to this article was reported.

\section{ACKNOWLEDGMENT}

We thank Prof. Dr. Jan Reedijk (University of Leiden), an international expert in platinum chemistry, for critical reading of the manuscript.

\section{REFERENCES}

1. Senter PD, Sievers EL. The discovery and development of brentuximab vedotin for use in relapsed Hodgkin lymphoma and systemic anaplastic large cell lymphoma. Nat Biotechnol. 2012;30:631-637.

2. Lambert JM, Chari RVJ. Ado-trastuzumab emtansine (T-DM1): an antibody-drug conjugate (ADC) for HER2-positive breast cancer. J Med Chem. 2014;57:6949-6964.

3. Lamb YN. Inotuzumab ozogamicin: first global approval. Drugs. 2017;77:1603-1610.

4. Drake PM, Rabuka D. Recent developments in ADC technology: preclinical studies signal future clinical trends. BioDrugs. 2017;31:521-531.

5. Beck A, Goetsch L, Dumontet C, Corvaïa N. Strategies and challenges for the next generation of antibody-drug conjugates. Nat Rev Drug Discov. 2017;16:315-337.

6. Chari RVJ, Miller ML, Widdison WC. Antibody-drug conjugates: an emerging concept in cancer therapy. Angew Chem Int Ed Engl. 2014;53:3796-3827.

7. Ducry L, Stump B. Antibody-drug conjugates: linking cytotoxic payloads to monoclonal antibodies. Bioconjug Chem. 2010;21:5-13.

8. Polakis P. Antibody drug conjugates for cancer therapy. Pharmacol Rev. 2016; $68: 3-19$.

9. Tsuchikama K, An Z. Antibody-drug conjugates: recent advances in conjugation and linker chemistries. Protein Cell. 2018;9:33-46.

10. Girish S, Gupta M, Wang B, et al. Clinical pharmacology of trastuzumab emtansine (T-DM1): an antibody-drug conjugate in development for the treatment of HER2-positive cancer. Cancer Chemother Pharmacol. 2012;69:1229-1240.

11. Lyon RP, Setter JR, Bovee TD, et al. Self-hydrolyzing maleimides improve the stability and pharmacological properties of antibody-drug conjugates. Nat Biotechnol. 2014;32:1059-1062.

12. Hamblett KJ, Senter PD, Chace DF, et al. Effects of drug loading on the antitumor activity of a monoclonal antibody drug conjugate. Clin Cancer Res. 2004; 10:7063-7070.

13. Sun X, Ponte JF, Yoder NC, et al. Effects of drug-antibody ratio on pharmacokinetics, biodistribution, efficacy, and tolerability of antibody-maytansinoid conjugates. Bioconjug Chem. 2017;28:1371-1381.

14. Erickson HK, Lambert JM. ADME of antibody-maytansinoid conjugates. AAPS J. 2012;14:799-805.

15. Erickson HK, Park PU, Widdison WC, et al. Antibody-maytansinoid conjugates are activated in targeted cancer cells by lysosomal degradation and linkerdependent intracellular processing. Cancer Res. 2006;66:4426-4433.
16. Okeley NM, Miyamoto JB, Zhang X, et al. Intracellular activation of SGN-35, a potent anti-CD30 antibody-drug conjugate. Clin Cancer Res. 2010;16:888-897.

17. de Goeij BE, Lambert JM. New developments for antibody-drug conjugate-based therapeutic approaches. Curr Opin Immunol. 2016;40:14-23.

18. Dennler P, Fischer E, Schibli R. Antibody conjugates: from heterogeneous populations to defined reagents. Antibodies (Basel). 2015;4:197-224.

19. McCombs JR, Owen SC. Antibody drug conjugates: design and selection of linker, payload and conjugation chemistry. AAPS J. 2015;17:339-351.

20. Waalboer DCJ, Muns JA, Sijbrandi NJ, et al. Platinum(II) as bifunctional linker in antibody-drug conjugate formation: coupling of a 4-nitrobenzo-2-oxa-1,3-diazole fluorophore to trastuzumab as a model. ChemMedChem. 2015;10:797-803.

21. Sijbrandi NJ, Merkul E, Muns JA, et al. A novel platinum(II)-based bifunctional ADC linker benchmarked using ${ }^{89} \mathrm{Zr}$-Desferal and auristatin F-conjugated trastuzumab. Cancer Res. 2017;77:257-267.

22. Kozak KR, Tsai SP, Fourie-O'Donohue A, et al. Total antibody quantification for MMAE-conjugated antibody-drug conjugates: impact of assay format and reagents. Bioconjug Chem. 2013;24:772-779.

23. Gorovits B, Alley SC, Bilic S, et al. Bioanalysis of antibody-drug conjugates: American Association of Pharmaceutical Scientists antibody-drug conjugate working group position paper. Bioanalysis. 2013;5:997-1006.

24. Kraynov E, Kamath AV, Walles M, et al. Current approaches for absorption, distribution, metabolism, and excretion characterization of antibody-drug conjugates: an industry white paper. Drug Metab Dispos. 2016;44:617-623.

25. Bobály B, Fleury-Souverain S, Beck A, et al. Current possibilities of liquid chromatography for the characterization of antibody-drug conjugates. J Pharm Biomed Anal. 2018;147:493-505.

26. Van Dongen GAMS, Huisman MC, Boellaard R, et al. ${ }^{89} \mathrm{Zr}$-immuno-PET for imaging of long circulating drugs and disease targets: why, how and when to be applied? Q J Nucl Med Mol Imaging. 2015;59:18-38.

27. Jauw YWS, Menke-van der Houven van Oordt CW, Hoekstra OS, et al. Immunopositron emission tomography with zirconium-89-labeled monoclonal antibodies in oncology: what can we learn from initial clinical trials? Front Pharmacol. 2016;7:131.

28. Cohen R, Vugts DJ, Visser GWM, et al. Development of novel ADCs: conjugation of tubulysin analogues to trastuzumab monitored by dual radiolabeling. Cancer Res. 2014;74:5700-5710.

29. Verel I, Visser GWM, Boellaard R, et al. ${ }^{89} \mathrm{Zr}$ immuno-PET: comprehensive procedures for the production of ${ }^{89} \mathrm{Zr}$-labeled monoclonal antibodies. $\mathrm{J} \mathrm{Nucl}$ Med. 2003;44:1271-1281.

30. Vosjan MJWD, Perk LR, Visser GWM, et al. Conjugation and radiolabeling of monoclonal antibodies with zirconium-89 for PET imaging using the bifunctional chelate p-isothiocyanatobenzyl-desferrioxamine. Nat Protoc. 2010;5:739-743.

31. Aalbersberg EA, de Wit-van der Veen BJ, Zwaagstra O, Codée-van der Schilden K, Vegt E, Vogel WV. Preclinical imaging characteristics and quantification of platinum-195m SPECT. Eur J Nucl Med Mol Imaging. 2017;44:1347-1354.

32. Yardeni T, Eckhaus M, Morris HD, Huizing M, Hoogstraten-Miller S. Retroorbital injections in mice. Lab Anim (NY). 2011;40:155-160.

33. Vugts DJ, Klaver C, Sewing C, et al. Comparison of the octadentate bifunctional chelator DFO*- $p$ Phe-NCS and the clinically used hexadentate bifunctional chelator DFO-pPhe-NCS for ${ }^{89} \mathrm{Zr}$-immuno-PET. Eur J Nucl Med Mol Imaging. 2017;44:286-295.

34. van Gog FB, Visser GWM, Klok RP, et al. Monoclonal antibodies labeled with rhenium-186 using the MAG3 chelate: relationship between the number of chelated groups and biodistribution characteristics. J Nucl Med. 1996;37:352-362.

35. Roopenian DC, Akilesh S. FcRn: the neonatal Fc receptor comes of age. Nat Rev Immunol. 2007;7:715-725.

36. Raghavan M, Bonagura VR, Morrison SL, Bjorkman PJ. Analysis of the $\mathrm{pH}$ dependence of the neonatal $\mathrm{Fc}$ receptor/immunoglobulin $\mathrm{G}$ interaction using antibody and receptor variants. Biochemistry. 1995;34:14649-14657.

37. Lambert JM, Morris CQ. Antibody-drug conjugates (ADCs) for personalized treatment of solid tumors: a review. Adv Ther. 2017;34:1015-1035.

38. Terwisscha van Scheltinga AGT, Ogasawara A, Pacheco G, et al. Preclinical efficacy of an antibody-drug conjugate targeting mesothelin correlates with quantitative ${ }^{89} \mathrm{Zr}$-immunoPET. Mol Cancer Ther. 2017;16:134-142.

39. Gebhart G, Lamberts LE, Wimana Z, et al. Molecular imaging as a tool to investigate heterogeneity of advanced HER2-positive breast cancer and to predict patient outcome under trastuzumab emtansine (T-DM1): the ZEPHIR trial. Ann Oncol. 2016;27:619-624.

40. Williams S-P, Ogasawara A, Tinianow JN, et al. ImmunoPET helps predicting the efficacy of antibody-drug conjugates targeting TENB2 and STEAP1. Oncotarget. 2016;7:25103-25112.

41. Jacobson O, Li Q, Chen H, et al. PET-guided evaluation and optimization of internalized antibody-drug conjugates targeting erythropoietin-producing hepatoma A2 receptor. J Nucl Med. 2017;58:1838-1844. 